\title{
ASYMPTOTIC ENUMERATION OF PARTIAL ORDERS ON A FINITE SET
}

\author{
BY \\ D. J. KLEITMAN( ${ }^{1}$ ) AND B. L. ROTHSCHILD( ${ }^{2}$ )
}

ABSTRACT. By considering special cases, the number $P_{\boldsymbol{n}}$ of partially ordered sets on a set of $n$ elements is shown to be $(1+O(1 / n)) Q_{n}$, where $Q_{n}$ is the number of partially ordered sets in one of the special classes. The number $Q_{n}$ can be estimated, and we ultimately obtain

$$
P_{n}=\left(1+O\left(\frac{1}{n}\right)\right)\left(\sum_{i=1}^{n} \sum_{j=1}^{n-i}\left(\begin{array}{c}
n \\
i
\end{array}\right)\left(\begin{array}{c}
n-i \\
j
\end{array}\right)\left(2^{i}-1\right)^{j}\left(2^{j}-1\right)^{n-i-j}\right) .
$$

1. Introduction. It is known [7] that the logarithm (base 2, as all logarithms in this paper will be) of the number $P_{n}$ of partial orders (or equivalently of $T_{0}$ topologies) on a set of $n$ elements is $n^{2} / 4+o\left(n^{2}\right)$. In this paper we show that $P_{n}$ is asymptotically equal to $Q_{n}$, the number of partial orders in a certain special class which is characterized in a simple way. It will follow that $\log P_{n}=n^{2} / 4+$ $3 n / 2+O(\log n)$. An explicit asymptotic formula for $P_{n}$ will be given, but it is a bit messy.

In [5] the number $G_{n}$ of "graded" partially ordered sets is enumerated. Since the partial orders counted by $Q_{n}$ turn out to be graded, the number arrived at in [5] is also asymptotically equal to $P_{n}$. The computation of $G_{n}[5]$, then, is asymptotically applicable to $P_{n}$.

The methods used here are similar to those used in [7] for obtaining the asymptotic estimate for $\log P_{n}$, but here they are somewhat more delicate and more complicated. We use induction to show that $P_{n} \leqslant Q_{n}(1+O(1 / n))$, while $Q_{n} \leqslant P_{n}$ by definition. The proof is accomplished by obtaining all partial orders on $n+1$ elements from those on $n$ or fewer elements in certain specified ways. In each of these ways, except those corresponding to $Q_{n+1}$, we obtain only an asymptotically negligible number of partial orders.

The partial orders corresponding to $Q_{n}$ can be described as follows. They consist of three sets $L_{1}, L_{2}, L_{3}$ with $\left|L_{1}\right|,\left|L_{3}\right|=n / 4+o(n),\left|L_{2}\right|=n / 2+o(n)$. Each element in $L_{i}$ "covers" only elements in $L_{i-1}$ (see below for definitions). And finally, each element behaves in the "average" way. That is, each element in

Received by the editors November 2, 1973 and, in revised form, January $21,1974$. AMS (MOS) subject classifications (1970). Primary 05A 15.

(1) Work supported in part by ONR Grant 0014-67-A-0204-0063.

(2) Work supported in part by NSF Grant GP-33580X. 
$L_{i}$ is covered by (asymptotically) half of those in $L_{i+1}$ and covers half of those in $L_{i-1}$.

2. Terminology. We represent a partial order $\boldsymbol{P}$ on a set of $n$ elements by its unique Hasse diagram, also denoted by $P$. This is a directed graph with the elements of $P$ as vertices and a single directed edge from $a$ to $b$ if and only if $a$ covers $b$ in $P$ ( $a$ covers $b$ if $a>b$ and $a>c \geqslant b$ implies $c=b$ ). Distinct partial orders have distinct diagrams. Thus we let $P_{n}$ denote both the number of partial orders and the number of diagrams on $n$ elements, as directed graphs diagrams are characterized by the exclusion of two types of configurations. Namely, a directed graph is a diagram of a partial order if and only if it contains no set of (directed) edges $E_{1}, E_{2}, \cdots, E_{k}, E_{0}$ such that the terminal vertex of $E_{i}$ is the initial vertex of $E_{i+1}, 1 \leqslant i \leqslant k-1$, and such that $E_{0}$ is incident with both the initial vertex of $E_{1}$ and the terminal vertex of $E_{k}$ (in either direction). That is, diagrams contain no directed cycles and no cycles with all but one edge cyclically directed. In particular, diagrams contain no triangles. We say that two vertices $a$ and $b$ are adjacent or connected if there is an edge incident with both (that is, if $a$ covers $b$ or $b$ covers $a$ ). For any set $S$ of vertices, $C(S)$ will denote the set of all vertices adjacent to any vertex in $S$.

We define levels of a diagram $P$ as follows. Level 1 consists of all minimal vertices of $P$ (vertices covering no other vertex). For each $j$, level $j$ is the set of minimal vertices obtained by deleting all vertices in levels $1, \cdots, j-1$. We observe that if $a$ is in level $i$ and $b$ is in level $j$, and if $i<j$, then either $a<b$ or $a$ and $b$ are incomparable. Two vertices in the same level are necessarily incomparable.

A diagram $P$ is bipartite if there are two parts $A$ and $B$ such that every edge of $P$ connects a vertex in $A$ with one in $B$, and $A \cap B=\varnothing$.

Consider a diagram $P$. Let $S$ be a subset of $V$, the vertex set of $P$. Then $P-S$ denotes the diagram obtained from $P$ by deleting all vertices of $S$ and all edges incident to any of them. (The resulting diagram is not necessarily the same as the diagram for the partially ordered set with elements $V-S$ and order induced from $P$.) The vertices of $P-S$ may be contained in the same level of $P-S$ as they were in $P$, or they may be contained in lower levels than they were in $P$. Let $P$ have $n$ vertices. Then a vertex $v$ in $P$ is called good if the number of vertices in lower levels in $P-\{v\}$ than in $P$ is less than $n^{1 / 2}$. Similarly a set $\left\{v_{1}, \cdots, v_{k}\right\}$ of vertices is good if deleting it affects the levels of fewer than $n^{1 / 2}$ other vertices. Every subset of a good set is good. Clearly no vertex can have its level affected by the deletion of vertices in higher levels. Also, if $v_{1}$ and $v_{2}$ are in the same level of $P$, and $S_{1}$ is the set of vertices with levels affected by deleting $v_{1}$, and $S_{2}$ by deleting $v_{2}$, 
then $S_{1} \cap S_{2}=\varnothing$. There can therefore be at most $n^{1 / 2}$ vertices in any single level which are not good. We call any vertex or set of vertices which is not good, bad.

3. Statement of lemma and theorem. Since the several cases of the lemma are rather technical, we will first state the lemma here. Then, in the next two sections, we will use it to prove the theorem. Finally, we will prove the lemma at the end.

In what follows, let $m$ be an arbitrary positive integer, and let $V$ be a set of $m+1$ vertices. We define a $(v, Q)$-set in a diagram $P$ on the set $V$ of vertices to be a good vertex $v$ adjacent to a set $Q$ of $\left[\mathrm{m}^{1 / 2}\right]$ vertices, either all covering $v$ or all covered by $v$, such that the level of each element of $Q$ is not affected by the deletion of $v$. We write $P=S_{1} \vee S_{2} \vee \cdots \vee S_{k}$ for a diagram $P$ to indicate that $V=S_{1} \cup S_{2} \cup \cdots \cup S_{k}, S_{i} \cap S_{j}=\varnothing$ if $i \neq j$, and vertices of $S_{i+1}$ can cover only vertices of $S_{i}, i=1,2, \cdots, k-1$. (The $S_{i}$ are not necessarily the levels of $P$. Consider for instance the diagram $P_{0}$ with no edges. Any partition of the vertices $S_{1}, \cdots, S_{k}$ satisfies $P=S_{1} \vee \cdots \vee S_{k}$, even though there is only one level.)

We consider the following classes of diagrams on $V$ :

$A(V)$ : Diagrams with some vertex $v$ adjacent to at most $m / 64$ other vertices. Let $A_{m+1}=|A(V)|$.

$B(V)$ : Diagrams with a $(v, Q)$-set with $|C(Q)| \geqslant m\left(1+m^{-3 / 8}\right) / 2$. Let $B_{m+1}=|B(V)|$.

$D(V)$ : Diagrams with a $(v, Q)$-set with $|C(Q)| \leqslant m\left(1-m^{-3 / 8}\right) / 2$. Let $D_{m+1}=|D(V)|$.

$E(V)$ : Diagrams with at least 30 nonempty levels. Let $E_{m+1}=|E(V)|$.

$F(V)$ : Diagrams with a $(v, Q)$-set with $|C(Q)|>m\left(1-m^{-3 / 8}\right) / 2$, such that the smallest set $R$ of vertices incident with every edge connecting two vertices in $V-(\{v\} \cup C(Q))$ satisfies $|R| \geqslant 2 m^{3 / 4}$. Let $F_{m+1}=|F(V)|$.

$G(V)$ : Diagrams not in $E(V)$ with a $(v, Q)$-set satisfying $m\left(1-m^{-3 / 8}\right) / 2<$ $|C(Q)|<m\left(1+m^{-3 / 8}\right) / 2$; with a set $R$ of at most $2 m^{3 / 4}$ vertices the deletion of which leaves no edge connecting two vertices of $V-(\{v\} \cup C(Q) \cup R)$; and such that the smallest set $S$ of vertices incident with all edges of $C(Q)$ satisfies $|S| \geqslant 2 m^{7 / 8}$. Let $G_{m+1}=|G(V)|$.

$H(V)$ : Diagrams $P$ satisfying the following properties: There is a set $T_{0}$ of at most $3 m^{7 / 8}$ vertices such that $P-T_{0}$ is bipartite; the parts $U$ and $W$ of $P-T_{0}$ have at least $m / 2-3 m^{7 / 8}$ and at most $m / 2+3 m^{7 / 8}$ vertices; $P-T_{0}$ has at most 29 levels, $L_{1}, L_{2}, \cdots, L_{k}, k \leqslant 29$; there is some level $L_{i}$ and a set $\{x, y\}$ in $P-T_{0}$ such that $\{x, y\}$ is a good set in $P-T_{0}$ and $L_{i} \cap C(x) \cap C(y)=\varnothing$; 
either $\left|L_{i} \cap U\right| \geqslant m^{15 / 16}$ and $\{x, y\} \subseteq W$, or $\left|L_{i} \cap W\right| \geqslant m^{15 / 16}$ and $\{x, y\} \subseteq U$. Let $H_{m+1}=|H(V)|$.

$I(V)$ : Diagrams $P$ not in $A(V)$ with a set $T$ of at most $10^{2} m^{15 / 16}$ vertices satisfying the following properties: $P-T=L_{1} \vee L_{2} \vee L_{3}$ where $L_{1}, L_{2}, L_{3}$ are the levels of $P-T$ ( $L_{3}$ possibly empty); both $\left|L_{1} \cup L_{3}\right|$ and $\left|L_{2}\right|$ are between $m / 2-10^{2} m^{15 / 16}$ and $m / 2+10^{2} m^{15 / 16}$; every two vertices in $W=L_{2}$ have a common adjacent vertex in $U=L_{1} \cup L_{3}$, and vice versa; there is a $t \in T$ adjacent both to a vertex $x$ in $L_{1} \cup L_{3}$ and a vertex $y \in L_{2}$. Let $I_{m+1}=|I(V)|$.

$J(V)$ : Diagrams $P$ with a set $T$ of at most $10^{2} \mathrm{~m}^{15 / 16}$ vertices satisfying the following properties: $P-T$ is a three level bipartite diagram with parts $U$ and $W$; $m / 2-10^{2} m^{15 / 16}<|U|<m / 2+10^{2} m^{15 / 16}$ and similarly for $|W|$; every two vertices in $U$ (resp. $W$ ) have a common adjacent vertex in $W$ (resp. $U$ ); there are two adjacent vertices $x, y$ in $T$ with $C(x)$ and $C(y)$ either both disjoint from $U$, or both disjoint from $W$. Let $J_{m+1}=|J(V)|$.

$K(V)$ : Bipartite diagrams $P$ not in $A(V)$ with a subset $T$ of at most $10^{2} m^{15 / 16}$ vertices satisfying the following: $P-T$ is a three level biparitite diagram with levels $L_{1}, L_{2}, L_{3}$, and parts $U=L_{1} \cup L_{3}, W=L_{2} ;|U|$ and $|W|$ are between $m / 2-10^{2} m^{15 / 16}$ and $m / 2+10^{2} m^{15 / 16}$; for each $t \in T$ and each $i$ either no vertex in $L_{i}$ covers $t$ or no vertex in $L_{i}$ is covered by $t$; there are vertices $x$ and $y$ in $T$ such that $C(x)-T \subseteq L_{1} \cup L_{3}, C(y)-T \subseteq L_{2}$ and no vertex of $C(x)-T$ is adjacent to any vertex of $C(y)-T$. Let $K_{m+1}=|K(V)|$.

$L(V)$ : Diagrams $P$ with a subset $T$ of at most $10^{2} \mathrm{~m}^{15 / 16}$ vertices such that $P-T$ has three levels, $L_{1}, L_{2}, L_{3}$, is bipartite, $m / 2-10^{2} m^{15 / 16}<\left|L_{2}\right|<m / 2+$ $10^{2} m^{15 / 16}$, and such that either there is an $x$ in $T$ covered only by vertices in $L_{1} \cup T$ and covering none, with $\left|L_{1}\right| \leqslant m / 2-m^{31 / 32}$, or there is an $x$ in $T$ covering only vertices in $L_{3} \cup T$ and covered by none, with $\left|L_{3}\right| \leqslant m / 2-m^{31 / 32}$. Let $L_{m+1}=|L(V)|$.

$M(V)$ : Diagrams $P$ such that $P=S_{1} \vee S_{2} \vee S_{3} \vee S_{4}$ and $\left|S_{2}\right|$ and $\left|S_{3}\right|$ are at least $m / 2-m^{31 / 32}$. Let $M_{m+1}=|M(V)|$.

$N(V)$ : Diagrams $P=S_{1} \vee S_{2} \vee S_{3}$ where at least one of the following inequalities does not hold: $(m+1) / 2-\log m<\left|S_{2}\right|<(m+1) / 2+\log m$, and for $i=1,3,(m+1) / 4-m^{1 / 2} \log m<\left|S_{i}\right|<(m+1) / 4+m^{1 / 2} \log m$. Let $N_{n+1}=|N(V)|$.

$X(V)$ : Diagrams $P=S_{1} \vee S_{2} \vee S_{3}$ which are not in $N(V)$. Let $X_{n+1}=$ $|X(V)|$

$O(V)$ : Diagrams in $X(V)$ with not all of the following inequalities valid: $(m+1) / 4-m^{7 / 8}<\left|C(v) \cap S_{2}\right|<(m+1) / 4+m^{7 / 8}$, for all $v$ in $S_{1} \cup S_{3}$, and $(m+1) / 8-m^{7 / 8}<\left|C(v) \cap S_{i}\right|<(m+1) / 8+m^{7 / 8}$, for $i=1,3$ and all $v$ in $S_{2}$. Let $O_{m+1}=|O(V)|$. 
$Q(V)$ : Diagrams in $X(V)-O(V)$. That is, each diagram $P$ in $Q(V)$ satisfies $P=L_{1} \vee L_{2} \vee L_{3}$, where $L_{1}, L_{2}, L_{3}$ are the levels of $P,(m+1) / 2-\log m<$ $\left|L_{2}\right|<(m+1) / 2+\log m,(m+1) / 4-m^{1 / 2} \log m<\left|L_{i}\right|<(m+1) / 4+$ $m^{1 / 2} \log m$ for $i=1,3$, and for each $u \in L_{1} \cup L_{3}$ and $w \in L_{2},(m+1) / 4-$ $m^{7 / 8}<\left|C(u) \cap L_{2}\right|<(m+1) / 4+m^{7 / 8}$, and $(m+1) / 8-m^{7 / 8}<\left|C(w) \cap L_{i}\right|$ $<(m+1) / 8+m^{7 / 8}, i=1,3$. Let $Q_{m+1}=|Q(V)|$.

LEMMA. There is a number $v$ such that for $n \geqslant v$ all of the following inequalities hold:

(1) $\log \left(A_{n+1} / P_{n}\right)<n / 4$,

(2) $\log \left(B_{n+1} / P_{n}\right)<n / 2-n^{5 / 8} / 4$,

(3) $\log \left(D_{n+1} / P_{n-\left[n^{1 / 2}\right]}\right)<n^{3 / 2} / 2-n^{9 / 8} / 4$,

(4) $\log \left(E_{n+1} / P_{n-29}\right)<9 n$,

(5) $\log \left(F_{n+1} / P_{n}\right)<n / 2-n^{3 / 4} / 5$,

(6) $\log \left(G_{n+1} / P_{n-1}\right)<n-n^{7 / 8} / 5$,

(7) $\log \left(H_{n+1} / P_{n-1}\right)<n-n^{15 / 16 / 4}$,

(8) $\log \left(I_{n+1} / P_{n}\right)<n / 2-n / 300$,

(9) $\log \left(J_{n+1} / P_{n-1}\right)<7 n / 8$,

(10) $\log \left(K_{n+1} / P_{n-1}\right)<99 n / 100$,

(11) $\log \left(L_{n+1} / P_{n}\right)<n / 2-n^{31 / 32 / 2}$,

(12) $\log \left(M_{n+1} / X_{n+1}\right)<-n / 4$,

(13) $\log \left(N_{n+1} / X_{n+1}\right)<-(\log n)^{2} / 6$,

(14) $n^{2} / 4+3 n / 2-3 \log n<\log X_{n}<n^{2} / 4+3 n / 2+\log n$,

(15) $\log \left(O_{n+1} / P_{n}\right)<n / 2-n^{3 / 4} / 10$.

THEOREM. $P_{n}=(1+O(1 / n)) Q_{n}$.

Corollary. $P_{n}=(1+O(1 / n)) f(n)$, where

$$
f(n)=\sum_{i=1}^{n}\left(\begin{array}{c}
n \\
i
\end{array}\right) \sum_{j=1}^{n-i}\left(\begin{array}{c}
n-i \\
j
\end{array}\right)\left(2^{j}-1\right)^{i}\left(2^{i}-1\right)^{n-i-i}
$$

The proof of the corollary is given at the end of the paper, after the proof of the lemma (\$7).

4. Proof of theorem. I. The proof is given in two parts. In part I we show that the classes $A(V)$ through $N(V), O(V)$ and $Q(V)$ are exhaustive. In part II we show that all classes except $Q(V)$ and $X(V)$ are negligible.

In the remainder of this paper we will adopt the convention that any inequality or other statement about functions of $n$ will be meant to be true only for all $n$ sufficiently large, where how large depends on the statement. This will 
be a convenience since there are so many such statements below.

Let $P$ be a diagram on the set $V$ of $n+1$ vertices, and let $P$ be in none of the classes described above except possibly $X(V)$ or $Q(V)$. In what follows we shall say "by $A$ ", "by $G$ ", etc., to mean "since $P$ is not in $A(V)$ ", "since $P$ is not in $G(V)$ ", etc., respectively.

By $E, P$ has at most 29 levels, and hence one level has at least $n / 29$ vertices. Of these we know that at most $n^{1 / 2}$ are bad. There must be a good vertex in this level, call it $v$. By $A, v$ must be adjacent to at least $n / 64$ other vertices, and since $v$ is good, at most $n^{1 / 2}$ of these have their levels affected by the deletion of $v$. Of the remaining vertices (at least $n / 64-n^{1 / 2}>2 n^{1 / 2}$ of them) adjacent to $v, v$ either covers or is covered by all vertices in a set $Q$ of $\left[n^{1 / 2}\right]$ vertices. Thus we have a $(v, Q)$-set in $P$.

By $B$ and $D,|C(Q)|$ must be between $n\left(1-n^{-3 / 8}\right) / 2$ and $n\left(1+n^{-3 / 8}\right) / 2$. By $F$ and $G$ there are sets $R$ and $S$ with $|R|<2 n^{3 / 4}$ and $|S|<2 n^{7 / 8}$ such that $P-(R \cup S)$ has no edges between two vertices of $V-(\{v\} \cup C(Q) \cup R \cup S)=$ $W^{\prime}$, or between two vertices of $(C(Q) \cup\{v\})-(R \cup S)=U^{\prime}$. Thus $P-(R \cup S)$ is bipartite with parts $U^{\prime}$ and $W^{\prime}$ and at most 29 levels. $|R \cup S|<3 n^{7 / 8}$.

Suppose there is a pair $x_{1}, y_{1}$ of vertices such that $\left\{x_{1}, y_{1}\right\}$ is a bad set in $P-(R \cup S)$. Then consider $P-\left(R \cup S \cup\left\{x_{1}, y_{1}\right\}\right)$. At least $\left(n-3 n^{7 / 8}\right)^{1 / 2}$ of the vertices of $P-\left(R \cup S \cup\left\{x_{1}, y_{1}\right\}\right)$ are in lower levels than in $P-(R \cup S)$. Now suppose $\left\{x_{2}, y_{2}\right\}$ is bad in $P-\left(R \cup S \cup\left\{x_{1}, y_{1}\right\}\right)$. Consider $P-$ $\left(R \cup S \cup\left\{x_{1}, y_{1}, x_{2}, y_{2}\right\}\right)$. At least $\left(n-3 n^{7 / 8}\right)^{1 / 2}$ vertices in $P-(R \cup S \cup$ $\left.\left\{x_{1}, y_{1}, x_{2}, y_{2}\right\}\right)$ are in lower levels than in $P-\left(R \cup S \cup\left\{x_{1}, y_{1}\right\}\right)$. We continue this process one step at a time, choosing $x_{1}, y_{1}, x_{2}, y_{2}, \cdots, x_{m}, y_{m}$ with $\left\{x_{j}, y_{j}\right\}$ a bad set in $P-\left(R \cup S \cup\left\{x_{1}, y_{1}, \cdots, x_{j-1}, y_{j-1}\right\}\right)$. We proceed until we can choose no more bad pairs $\{x, y\}$. For $j \leqslant n^{3 / 4}$, each step decreases the levels of at least $\left(n-3 n^{7 / 8}\right)^{1 / 2}$ vertices, since $\left|R \cup S \cup\left\{x_{1}, y_{1}, \cdots, x_{j-1}, y_{j-1}\right\}\right|$ $<3 n^{7 / 8}$. Since each vertex can decrease its level at most 28 times during this process, and since $n^{3 / 4}\left(n-3 n^{7 / 8}\right)^{1 / 2}>28(n+1)$, the total number $m$ of steps before the process stops is at most $n^{3 / 4}$.

Let $T_{0}=R \cup S \cup\left\{x_{1}, y_{1}, \cdots, x_{m}, y_{m}\right\}$. Then $P-T_{0}$ is bipartite with parts $U^{\prime}-T_{0}$ and $W^{\prime}-T_{0}$, where $\left|T_{0}\right|<3 n^{7 / 8}$, and $\left|W^{\prime}-T_{0}\right|$ and $\left|U^{\prime}-T_{0}\right|$ are between $n / 2-3 n^{7 / 8}$ and $n / 2+3 n^{7 / 8}$. Let the levels of $P-T_{0}$ be $L_{1}, L_{2}$, $\because \cdots, L_{k}, k \leqslant 29$. Let $h$ be the largest value of $j$ such that either $\left|L_{j} \cap U^{\prime}\right| \geqslant$ $n^{15 / 16}$ or $\left|L_{j} \cap W^{\prime}\right| \geqslant n^{15 / 16}$, say $\left|L_{h} \cap U^{\prime}\right| \geqslant n^{15 / 16}$.

Then we claim $h \leqslant 3$. For suppose $h \geqslant 4$. Let $u>x>z>y$ be in levels $h, h-1, h-2, h-3$, respectively, with $u \in L_{h} \cap U^{\prime}$. Then $x, y \in W^{\prime}$ and $z \in U^{\prime}$, since $P-T_{0}$ is bipartite. Now by $H, C(x) \cap C(y) \cap L_{h} \neq \varnothing$; let $u^{\prime} \in C(x) \cap C(y) \cap L_{i}$. Then $u^{\prime}>x>z>y$, and $u^{\prime}$ also covers $y$, since it is 
adjacent and in a higher level. But then $u^{\prime}, x, z, y$ form an excluded configuration, a contradiction. Hence $h \leqslant 3$.

For $i=1,2,3$, we claim that if $\left|L_{i} \cap U^{\prime}\right| \geqslant n^{15 / 16}$ (respectively $\left|L_{i} \cap W^{\prime}\right|$ $\geqslant n^{15 / 16}$ ), then $L_{i} \cap W^{\prime}=\varnothing$ (respectively $L_{i} \cap U^{\prime}=\varnothing$ ). For let $x \in L_{i} \cap W^{\prime}$ (respectively $x \in L_{i} \cap U^{\prime}$ ). By $H$, as above, $x$ must be adjacent to some vertex in $L_{i} \cap U^{\prime}$ (respectively $L_{i} \cap W^{\prime}$ ), a contradiction since no two vertices in $L_{i}$ can be adjacent. Thus if $\left|L_{i}\right| \geqslant 2 n^{15 / 16}, L_{i}$ must be entirely in $U^{\prime}$ or entirely in $W^{\prime}$.

Since $\left|U^{\prime}-T_{0}\right|$ and $\left|W^{\prime}-T_{0}\right|$ are both at least $n / 2-3 n^{7 / 8}$, and since $\left|L_{j}\right|<2 n^{15 / 16}$ for $j>3$, we must have at least one of $L_{1}, L_{2}, L_{3}$ entirely in $U^{\prime}$ with at least $n^{15 / 16}$ vertices, and one entirely in $W^{\prime}$ with at least $n^{15 / 16}$ vertices. In particular, $L_{2}$ is entirely in $W^{\prime}$ or entirely in $U^{\prime}$. For if not, $L_{1}$ would be entirely in $U^{\prime}$ or entirely in $W^{\prime}$. But then $L_{2}$ would be entirely in $W^{\prime}$ or entirely in $U^{\prime}$, since every vertex in $L_{2}$ is adjacent to some vertex of $L_{1}$. Similarly, since $L_{2} \subseteq W^{\prime}$ or $L_{2} \subseteq U^{\prime}$, we must have $L_{3} \subseteq U^{\prime}$ or $L_{3} \subseteq W^{\prime}$. Now either $\left|L_{1}\right|<$ $2 n^{15 / 16}$ or $L_{1} \subseteq U^{\prime}$ or $L_{1} \subseteq W^{\prime}$. Let $T=T_{0} \cup \bigcup_{j>3} L_{j} \cup L_{1}$ if $\left|L_{1}\right|<2 n^{15 / 16}$, and $T=T_{0} \cup \cup_{j>3} L_{j}$ if $\left|L_{1}\right| \geqslant 2 n^{15 / 16}$ (and thus $L_{1} \subseteq U^{\prime}$ or $L_{1} \subseteq W^{\prime}$ ).

$|T|<60 n^{15 / 16}$, and $P-T$ is bipartite with parts $U=U^{\prime}-T$ and $W=$ $W^{\prime}-T$, with $|U|$ and $|W|$ between $n / 2-60 n^{15 / 16}$ and $n / 2+60 n^{15 / 16} . P-T$ has two or three levels, $L_{1}, L_{2}, L_{3}$ (where $L_{3}=\varnothing$ in the two level case). Finally $U=L_{1} \cup L_{3}, W=L_{2}$, or $U=L_{2}, W=L_{1} \cup L_{3}$. We assume, without loss of generality, $L_{1} \cup L_{3}=U, L_{2}=W$. By construction of $U$ and $W$, and by $H$, every two vertices of $U$ are adjacent to a common vertex of $W$, and vice versa. Also $\left|L_{1}\right| \geqslant 2 n^{15 / 16}$.

By $I$, no vertex in $T$ is adjacent both to vertices of $W$ and $U$. Hence $T$ is divided into two subsets, $T_{U}$ and $T_{W}$, where $C\left(T_{W}\right) \cap W=\varnothing . C\left(T_{U}\right) \cap U=\varnothing$. By $J$, no two vertices of $T_{U}$ are adjacent, and no two vertices of $T_{W}$ are adjacent. Thus $P$ itself is bipartite with parts $U \cup T_{U}$ and $W \cup T_{W}$.

Suppose $t \in T, x, y \in L_{i}, i=1,2$ or 3 , and $t$ is adjacent to $x$ and to $y$. Then either $t$ covers both $x$ and $y$ or $t$ is covered by both. For if $t$ covers $x$ and $y$ covers $t$, by construction of $U$ and $W$ we can let $v$ be a vertex in $L_{i-1}$ or $L_{i+1}$ to which both $x$ and $y$ are adjacent. Then $t, x, y, v$ form an excluded configuration, a contradiction.

Suppose $t \in T$ is adjacent to vertices in $x$ and $y$ in different levels of $P-T$. Then these levels must be $L_{1}$ and $L_{3}$ (by $I$ ). $x$ and $y$ are adjacent to a common vertex $v$ in $L_{2}$. Let $x$ be the vertex in $L_{1}, y \in L_{3}$. Then $y$ covers $v, v$ covers $x$, and we must have that $y$ covers $t$ and $t$ covers $x$, or else $x, y, v, t$ form an excluded configuration. So if $t$ is adjacent to vertices in $L_{1}$ and $L_{3}$, it covers those in $L_{1}$ and is covered by those in $L_{3}$.

All vertices of $T$, therefore, are in one of the following subsets: 
$T_{i}^{+}=\left\{t \mid t\right.$ covers only vertices in $L_{i}$ and is covered by none in $\left.V-T\right\}$,

$T_{i}^{-}=\left\{t \mid t\right.$ is covered only by vertices in $L_{i}$ and covers none in $\left.V-T\right\}$

$$
\text { for } i=1,2,3 \text {, }
$$

$T_{2}^{0}=\left\{t \mid t\right.$ covers vertices in $L_{1}$ and is covered by vertices in $L_{3}$, and is adjacent to no others in $V-T$ \}.

We now claim that

$$
P=\left(T_{1}^{-}\right) \vee\left(L_{1} \cup T_{2}^{-}\right) \vee\left(L_{2} \cup T_{1}^{+} \cup T_{3}^{-} \cup T_{2}^{0}\right) \vee\left(L_{3} \cup T_{2}^{+}\right) \vee\left(T_{3}^{+}\right) \text {. }
$$

To show this we must show that no vertex of $T_{3}^{+}$is adjacent to any in $T_{2}$, no vertex of $T_{1}^{-}$is adjacent to any in $T_{2}^{+}$, and all other adjacencies are in the "right direction." (We already know that there are no edges between two vertices of $T_{1}^{-} \cup T_{1}^{+} \cup T_{3}^{-} \cup T_{3}^{+} \cup T_{2}^{0}$ or between two vertices of $T_{2}^{-} \cup T_{2}^{+}$.)

If $x \in T_{3}^{+}, y \in T_{2}^{-}$, are adjacent, there can be no edge between $C(x)-T$ and $C(y)-T$ or we would have an excluded configuration. Thus by $K, x$ and $y$ cannot be adjacent. Similarly, $x \in T_{1}^{-}$and $y \in T_{2}^{+}$cannot be adjacent.

Now suppose $x \in T_{i}^{-}, y \in T_{i+1}^{-}, i=1$ or 2 . If $x$ covers $y, C(x)-T$ and $C(y)-T$ must have no edge between them, or an excluded configuration would result. Hence, by $K, x$ cannot cover $y$. Similarly, if $x \in T_{i}^{+}, y \in T_{i+1}^{-}, i=1$ or 2 , then $y$ cannot cover $x$.

Finally, if $x \in T_{2}^{-}$and $y \in T_{2}^{+}$(or $y \in T_{2}^{-}, x \in T_{2}^{+}$, resp.) and if $x$ covers $y$ (respectively $x$ is covered by $y$ ), then no vertex of $C(x)-T$ is adjacent to any vertex of $C(y)-T$, or an excluded configuration would result. Thus, by $K, x$ cannot cover $y$ (respectively $y$ cannot cover $x$ ). This completes the elimination of all possible connections which would contradict the claim. Thus we have shown that

$$
P=\left(T_{1}^{-}\right) \vee\left(T_{2}^{-} \cup L_{1}\right) \vee\left(L_{2} \cup T_{1}^{+} \cup T_{3}^{-} \cup T_{2}^{0}\right) \vee\left(L_{2} \cup T_{2}^{+}\right) \vee\left(T_{3}^{+}\right) .
$$

(We recall that $L_{3}=\varnothing$ is possible here, in which case $T_{3}^{+}, T_{3}^{-}, T_{2}^{0}$ are all empty as well.)

Since $\left|L_{2}\right| \geqslant n / 2-10^{2} n^{15 / 16}$, we must have $\left|L_{3}\right| \leqslant n / 2-n^{31 / 32}$ or $\left|L_{1}\right| \leqslant$ $n / 2-n^{31 / 32}$. Then by $L$, either $T_{1}^{-}=\varnothing$ or $T_{3}^{+}=\varnothing$, or both. But if $T_{1}^{-} \neq \varnothing$, by $L$ we must have $\left|L_{1}\right|>n / 2-n^{31 / 32}$; or if $T_{3}^{+} \neq \varnothing,\left|L_{3}\right|>n / 2-n^{31 / 32}$. By $M$, neither of these possibilities occurs. Hence $T_{1}^{-}=T_{3}^{+}=\varnothing$.

This gives us $P=S_{1} \vee S_{2} \vee S_{3}$, where $S_{1}=L_{1} \cup T_{2}^{-}, S_{2}=L_{2} \cup T_{2}^{0} \cup$ $T_{1}^{+} \cup T_{3}^{-}, S_{3}=L_{3} \cup T_{2}^{+}$. Finally, then, by $N$ and $O, P$ must be in $Q(V)$. In particular, since every vertex of $S_{2}$ covers some (at least $n / 8-n^{7 / 8}$ ) vertices of 
$S_{1}$, and every vertex of $S_{3}$ covers some (at least $n / 4-n^{7 / 8}$ ) vertices of $S_{2}$, the $S_{i}$ are in fact levels in $P$. Part I of the proof of the theorem is now complete.

5. Proof of theorem. II. We shall use the results of the last section, together with the lemma, to show that $P_{n} \leqslant(1+O(1 / n)) Q_{n}$. First we show that $P_{n} \leqslant(1+O(1 / n)) X_{n}$.

Let $\nu$ be the number guaranteed by the lemma, and let $N=\max \left(2 v, 10^{9}\right)$.

Let $C_{0}$ be a number large enough so that $P_{n} \leqslant\left(1+\left(C_{0}\right) / n\right) X_{n}$ for all $n \leqslant N$, and let $C=\max \left(C_{0}, 10^{9}\right)$. We claim that $P_{n} \leqslant(1+C / n) \dot{X}_{n}$ for all $n$.

The proof of this claim is by induction on $n$. For $n \leqslant N$ it is true by choice of $C$. We assume that it is true for all $n \leqslant m$, for some $m \geqslant N$, and show that $P_{m+1} \leqslant(1+C /(m+1)) X_{m+1}$ as well. Since, by the last section, we have $P_{m+1} \leqslant$ $A_{m+1}+B_{m+1}+D_{m+1}+\cdots+N_{m+1}+X_{m+1}$, we need only show that $\left(A_{m+1}+\cdots+N_{m+1}\right) /\left(X_{m+1}\right) \leqslant C /(m+1)$. To do this we shall employ the inequalities of the lemma to show that each of the terms $A_{m+1} /\left(X_{m+1}\right)$, $B_{m+1} /\left(X_{m+1}\right), \cdots, N_{m+1} /\left(X_{m+1}\right)$ is at most $1 / 13 \cdot C /(m+1)$ (there are 13 terms here). These arguments are all similar, and we illustrate a few typical ones.

(1) $\frac{A_{m+1}}{X_{m+1}}=\frac{A_{m+1}}{P_{m}} \frac{P_{m}}{X_{m}} \frac{X_{m}}{X_{m+1}}<2^{m / 4}(1+C / m) 2^{-m / 2+5 \log m}<\frac{C}{m+1} \cdot \frac{1}{13}$, $\frac{D_{m+1}}{X_{m+1}}=\frac{D_{m+1}}{P_{m-\left[m^{1 / 2}\right]}} \frac{P_{m-\left[m^{1 / 2}\right]}}{X_{m-\left[m^{1 / 2}\right]}} \frac{X_{m-\left[m^{1 / 2}\right]}}{X_{m-\left[m^{1 / 2}\right]}+1} \cdots \frac{X_{m}}{X_{m+1}}$

$$
\begin{aligned}
& <2^{m^{3 / 2} / 2-1 / 4 m^{9 / 8}}\left(1+\frac{C}{m-\left[m^{1 / 2}\right]}\right) 2^{-\left(m / 2-\left[m^{1 / 2}\right] / 2\right)\left(\left[m^{1 / 2}\right]+1\right)} \\
& <\frac{1}{13} \frac{C}{m+1},
\end{aligned}
$$

$$
\begin{aligned}
\frac{N_{m+1}}{X_{m+1}} & <2^{-(\log m)^{2 / 6}}, \text { by the lemma, } \\
& <\frac{1}{13} \frac{C}{m+1} .
\end{aligned}
$$

These and similar arguments, then, for the other cases complete the induction. We thus have $P_{n} \leqslant(1+C / n) X_{n}$ for all $n$.

By the lemma, $O_{n+1} / P_{n} \leqslant 2^{(n / 2)-(1 / 10) n 3 / 4}$ for $n \geqslant N_{0}$. Also, by the definitions of $O(V), X(V)$ and $Q(V)$, we have $X_{n+1}=O_{n+1}+Q_{n+1}$. These facts lead to 


$$
\begin{aligned}
P_{n+1} & \leqslant\left(1+\frac{C}{n+1}\right) X_{n+1}=\left(1+\frac{C}{n+1}\right) Q_{n+1} \frac{X_{n+1}}{Q_{n+1}} \\
& =\left(1+\frac{C}{n+1}\right) Q_{n+1}\left(1-\frac{O_{n+1}}{P_{n}} \frac{P_{n}}{X_{n}} \frac{X_{n}}{X_{n+1}}\right)^{-1} \\
& <\left(1+\frac{C}{n+1}\right) Q_{n+1}\left(1-2^{-(1 / 10) n^{3 / 4}}\right)^{-1} \\
& <\left(1+\frac{2 C}{n+1}\right) Q_{n+1} \text { for } n \text { sufficiently large. }
\end{aligned}
$$

This establishes $P_{n}=(1+O(1 / n)) Q_{n}$ and completes the proof of the theorem.

6. Proof of lemma. We let $V$ be a set of $n+1$ vertices. We recall our convention, that all statements in inequalities asserted below are meant to be valid only for $n$ sufficiently large. More specifically, for each statement below there is a number $N^{\prime}$ such that the statement is valid for $n \geqslant N^{\prime}$. We can then let $\nu$ be the maximum of all of these $N^{\prime}$. The numbered paragraphs below correspond to the numbered inequalities of the lemma.

For the sake of brevity, we include only a few of the cases in detail. The rest of them are represented only by the final inequalities of the arguments, from which one can obtain a hint as to the order in which things are constructed. We choose one simple case, and the most complicated ones to do in detail.

(1) This inequality is Lemma 2 of [7]. It is proved like those below.

(2) This and (3) below correspond to Lemmas 3 and 4 of [7].

We obtain all diagrams in $B(V)$ from diagrams on $n$ vertices by choosing $v \in V$, choosing a diagram on $V-\{v\}$, and then adjoining $v$ to the diagram so as to satisfy the conditions for $B(V)$. We will obtain upper bounds for the number of possible choices by counting some possibilities which cannot satisfy the conditions for $B(V)$ as well as all those that do. This is the general method used below, where instead of just choosing a single $v$, we may need to choose a subset $S \subset V$, a diagram on $V-S$, and then to adjoin $S$ to the diagram.

To obtain diagrams in $B(V)$, then, we choose $v \in V(n+1$ ways to choose $v$ ) and a diagram on $V-\{v\}$ (at most $P_{n}$ ways to do this). Now $v$ will be taken as the vertex of a $(v, Q)$-set.

To connect $v$, we first choose a level for $v$ to be in (at most $n+1$ possibilities). Then we choose $Q$ (at most ${ }_{n} C_{\left[n^{1 / 2}\right]}$ ways, where many of the sets of $\left[n^{1 / 2}\right]$ vertices in $V-\{v\}$ included in this number will not be valid candidates for $Q$, depending on which diagram was chosen for $V-\{v\})$. The directions of the connections between $v$ and $Q$ are now determined, because in order to be in 
$B(V)$, the levels of vertices in $Q$ must be unaffected by the addition of $v$. Hence $v$ covers all vertices of $Q$ if its level is higher than all of them, and is covered by all vertices if its level is lower. (One of these two possibilities must occur, or we would have an invalid choice for $Q$.)

Since $Q$ will satisfy conditions for $B(V)$, we have $|C(Q)| \geqslant n\left(1+n^{-3 / 8}\right) / 2$. Since there are no triangles, $v$ can be connected to at most $n\left(1-n^{-3 / 8}\right) / 2$ remaining vertices. Since $v$ must be a good vertex, at most $n^{1 / 2}$ of these vertices can have their levels affected by the addition of $v$. The vertices which can have their levels changed can be chosen in at most

$$
\sum_{i=0}^{\left[n^{1 / 2}\right]}\left(\begin{array}{c}
{\left[n\left(1-n^{-3 / 8}\right) / 2\right]} \\
i
\end{array}\right) \leqslant n(n / 2)^{n^{1 / 2}}
$$

ways. $v$ can then be connected to these vertices in at most $3^{n^{1 / 2}}$ ways (the 3 is for the choices: $v$ covers $x, x$ covers $v$, and $x$ and $v$ not connected). Finally, the other vertices to which $v$ can be connected can be chosen in at most $2^{n\left(1-n^{-3 / 8}\right) / 2}$ ways. The directions of these connections are determined by the levels relative to the level of $v$. All connections of $v$ are now completed. Multiplying all these numbers of possible choices gives the following:

$$
\begin{aligned}
\log \left(\frac{B_{n+1}}{P_{n}}\right) \leqslant & \log (n+1)+\log (n+1)+\log \left(\begin{array}{c}
n \\
{\left[n^{1 / 2}\right]}
\end{array}\right) \\
& +\log \left(n(n / 2)^{n^{1 / 2}}\right)+n^{1 / 2} \log 3+n\left(1-n^{-3 / 8}\right) / 2 \\
< & 2 \log (n+1)+n^{1 / 2} \log n+\log n+n^{1 / 2} \log n / 2 \\
& +n^{1 / 2} \log 3+n / 2-n^{5 / 8} / 2 \\
< & n / 2-n^{5 / 8} / 4 .
\end{aligned}
$$

This completes (2). We used one of two basic relations here, which will be used repeatedly below.

$$
\log \left(\begin{array}{c}
n \\
{\left[n^{\alpha}\right]}
\end{array}\right)<n^{\alpha} \log n
$$

The other is Stirling's formula or the normal approximation, which gives (recall, for $n$ sufficiently large, here depending on the $\beta$ ):

$$
\begin{array}{r}
\log \left(\begin{array}{c}
n \\
{\left[\alpha^{n}\right]}
\end{array}\right)<-n(\alpha \log \alpha+(1-\alpha) \log (1-\alpha)) \\
\quad \text { for } 0<\beta \leqslant \alpha \leqslant 1 / 2 \text {, and } \beta \text { fixed (independent of } n) .
\end{array}
$$


$\log \left(\begin{array}{c}n \\ {[n(1-\alpha) / 2]}\end{array}\right)<n-1 / 2 n \alpha^{2}$ for $0 \leqslant \alpha \leqslant \beta<1$, and $\beta$ fixed. $\log \left(\frac{D_{n+1}}{P_{n-\left[n^{1 / 2}\right]}}\right) \leqslant \log (n+1)+\log \left(\begin{array}{c}n \\ {\left[n^{1 / 2}\right]}\end{array}\right)+1$

$$
\begin{aligned}
& +\log \left(\frac{n}{2}\left(\begin{array}{c}
n \\
{[n / 2]}
\end{array}\right)\right)+\left(1+n^{1 / 2}\right)\left(n / 2-n^{5 / 8} / 2\right)+n \log 3 \\
& <n^{3 / 2} / 2-n^{9 / 8} / 4
\end{aligned}
$$

(4) $\quad \log \left(\frac{E_{n+1}}{P_{n-29}}\right) \leqslant \log \left(\begin{array}{c}n+1 \\ 30\end{array}\right)+\log (30 !)+(n-29) \log 496<9 n$.

(5) $\quad \log \left(\frac{F_{n+1}}{P_{n}}\right) \leqslant 3 n^{1 / 2} \log n+n\left(1+n^{-3 / 8}\right) / 2-2 n^{3 / 4}+n^{3 / 4} \log 3$

$$
<n / 2-n^{3 / 4} / 5
$$

(6) $\log \left(\frac{G_{n+1}}{P_{n-1}}\right)<15 n^{3 / 4} \log n+\frac{n}{2}+\frac{n}{2}-2 n^{7 / 8}+n^{7 / 8} \log 3<n-n^{7 / 8} / 5$.

$$
\log \frac{H_{n+1}}{P_{n-1}}<10 n^{7 / 8} \log n+n-2 h+h \log 3<n-n^{15 / 16} / 4
$$

(8) We obtain all diagrams in $I(V)$ by considering two subclasses $I^{\prime}(V)$ and $I^{\prime \prime}(V)$. $I^{\prime}(V)$ will be the class of diagrams in $I(V)$ such that $T$ contains a vertex $t$ and a set $S \subseteq C(t) \cap W$ (respectively, $C(t) \cap U)$ of $\left[n^{16 / 17}\right]$ vertices such that $C(S) \cap U$ (respectively, $C(S) \cap W)$ has at most $n / 2-n / 300$ vertices. $I^{\prime \prime}(V)=$ $I(V)-I^{\prime}(V)$.

We obtain diagrams in $I^{\prime}(V)$ by choosing $T, t, L_{1}, L_{2}$ (and $\left.L_{3}\right), S, C(S) \cap U$ (respectively $C(S) \cap W$ ) (there are at most $2^{6 n}$ ways to make these choices), and then connecting the vertices of $L_{1}, L_{2}, L_{3}$ and $T . T$ can be connected to $V$ in at most $3^{10^{2} n} 15 / 16_{n}$. This leaves the connections of $U$ to $W$ to be made. The directions of these connections are already determined by the choice of $L_{1}, L_{2}$ and $L_{3} . S$ can be connected to $U$ (respectively $W$ ), then, in at most

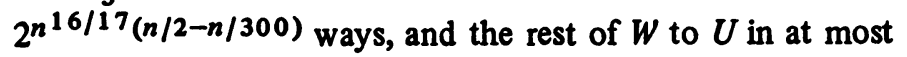

$$
2^{\left(n / 2+10^{2} n^{15 / 16}-n^{16 / 17}\right)\left(n / 2+10^{2} n^{15 / 16}\right)}
$$

ways. This gives: 


$$
\log \left|I^{\prime}(V)\right|<6 n+10^{2} n^{31 / 16} \log 3+n^{2} / 4-n^{33 / 17} / 400 .
$$

We know from [7], or trivially by direct observation, that $P_{n}>2^{n^{2} / 4}$ (also from paragraph (14) below). Thus

$$
\log \left|\ell^{\prime}(V)\right| / P_{n}<-n^{33 / 17} / 500 .
$$

We obtain diagrams in $I^{\prime \prime}(V)$ as follows: We choose $t(n+1$ ways) and a diagram on $V-\{t\}$ so that there is a set $T^{\prime}$ with $T=T^{\prime} \cup\{t\}$ satisfying conditions for $I(V)$ (at most $P_{n}$ ways, and at most

$$
n\left(\begin{array}{c}
n \\
{\left[10^{2} n^{15 / 16}\right]}
\end{array}\right)
$$

ways to choose $T^{\prime}$ ). Then, since every two vertices in $U$ (respectively, $W$ ) are adjacent to a common vertex of $W$ (respectively, $U$ ), $t$ can be connected to each level in only one direction or an excluded configuration results (at most $2^{3}$ choices for directions for $t$ ). $t$ can be connected to $T^{\prime}$ at most $3^{10^{2}} n^{15 / 16}$ ways. To satisfy the conditions on $I(V)$ there must be vertices $x \in U$ and $y \in W$ adjacent to $t$ (at most $n^{2}$ ways to choose $x$ and $y$, and 4 ways to connect them to $t$ ).

By $A, t$ must be adjacent to $n / 64$ other vertices at least, and of those to at least $n^{16 / 17}$ in $U$, or $n^{16 / 17}$ in $W$, say $W$. There are at most

$$
\left(\begin{array}{c}
{\left[n / 2+10^{2} n^{15 / 16}\right]} \\
{\left[n^{16 / 17}\right]}
\end{array}\right)
$$

ways to choose a set $S$ of $\left[n^{16 / 17}\right]$ vertices in $W$ to be included in $C(t) \cap W$. But by the conditions for $I^{\prime \prime}(V),|C(S) \cap U| \geqslant n / 2-n / 300 . x$ must thus be in $U-(C(S) \cap U)$ and $C(x) \cap W$ must be at least $n / 65$, by $A$. The remaining vertices of $C(t)$ must be chosen from $((W-S)-C(x)) \cup(U-C(S))$, of which there are at most

$$
\begin{gathered}
\left(n / 2+10^{2} n^{15 / 16}-\left[n^{16 / 17}\right]-n / 65+n / 2+10^{2} n^{15 / 16}-n / 2+n / 300\right) \\
<n / 2-n / 130 .
\end{gathered}
$$

Thus there are at most $2^{n / 2-n / 130}$ ways to complete the connections of $t$.

This all gives

$$
\log \left(\frac{\left|I^{n}(V)\right|}{P_{n}}\right)<6 n^{16 / 17} \log n+\frac{n}{2}-\frac{n}{130}<\frac{n}{2}-\frac{n}{200} .
$$

Thus

$$
\log \left(\frac{I_{n+1}}{P_{n}}\right)=\log \left(\frac{\left|I^{\prime}(V)\right|+\left|I^{n}(V)\right|}{P_{n}}\right)<\log \left(\frac{\left|I^{\prime \prime}(V)\right|}{P_{n}}+1\right)<\frac{n}{2}-\frac{n}{300} .
$$




$$
\log \left(\frac{J_{n+1}}{P_{n-1}}\right)<9 \cdot 10^{2} n^{15 / 16} \log n+\frac{n}{2} \log 3<\frac{7}{8} n .
$$

$$
\log \left(\frac{K_{n+1}}{P_{n-1}}\right)<10^{4} n^{15 / 16} \log n+\frac{n}{2}+\frac{n}{2}-\frac{n}{66}<\frac{99}{100} n
$$

(11) $\log \left(\frac{L_{n+1}}{P_{n}}\right)<\frac{n}{2}-n^{31 / 32}+5 \cdot 10^{2} n^{15 / 16} \log n<\frac{n}{2}-\frac{1}{2} n^{31 / 32}$.

(14) We now estimate $X_{n}$ so we can use it for $M_{n+1}$ and $N_{n+1}$. We obtain a lower bound for $X_{n}$ by considering the subclass consisting of all three level diagrams $P=L_{1} \vee L_{2} \vee L_{3}$ with $\left|L_{2}\right|=[n / 2],\left|L_{1}\right|=[n / 4],\left|L_{3}\right|=$ $n-[n / 2]-[n / 4]$. We can choose $L_{2}$ in $\left(\begin{array}{c}n \\ {[n / 2]}\end{array}\right)$ ways, and then $L_{1}$ in $\left(\begin{array}{c}n-[n / 2] \\ {[n / 4]}\end{array}\right)$. Each vertex in $L_{2}$ can be connected to $L_{1}$ in $2^{[n / 4]}-1$ ways. (The -1 is necessary because $L_{2}$ is a level and thus each vertex in $L_{2}$ must be adjacent to at least one in $L_{1}$.) Each vertex in $L_{3}$ can be connected to $L_{2}$ in $\left(2^{[n / 2]}-1\right)$ ways. Thus we can choose the edges of $P$ in $\left(2^{[n / 2]}-1\right)^{n-[n / 2]-[n / 4]}\left(2^{[n / 4]}-1\right)^{[n / 2]}$ ways. We only get diagrams counted by $X_{n}$ here, and we get no diagrams twice. Thus

$$
\begin{aligned}
\log X_{n}> & \log \left(\begin{array}{c}
n \\
{[n / 2]}
\end{array}\right)+\log \left(\begin{array}{c}
n-[n / 2] \\
{[n / 4]}
\end{array}\right)+[n / 2](n-[n / 2]-[n / 4]) \\
& +[n / 2][n / 4]+[n / 2] \log \left(1-1 / 2^{[n / 4]}\right) \\
& +(n-[n / 2]-[n / 4]) \log \left(1-1 / 2^{[n / 2]}\right) \\
> & \log \left(1 / n \cdot 2^{n}\right)+\log \left(1 / n \cdot 2^{n / 2}\right) \\
& +(n-[n / 2])[n / 2]-1>n^{2} / 4+3 n / 2-3 \log n .
\end{aligned}
$$

We find an upper bound for $X_{n}$ equally easily. Let $V^{\prime}$ be a set of $n$ elements. Diagrams in $X\left(V^{\prime}\right)$ are obtained as follows. We choose $S_{2}$ (at most $2^{n}$ ways); $S_{1}$ from $V-S_{2}$ (at most $2^{n / 2+\log n}$ ways); and connections from $S_{1} \cup S_{3}$ to $S_{2}$ (at most $2^{n^{2} / 4}$ ways). This gives

$$
\log \left(X_{n}\right)<n^{2} / 4+3 n / 2+\log n \text {. }
$$

Together with the lower bound for $X_{n+1}$ we get $\log \left(X_{n} / X_{n+1}\right)<-n / 2+5 \log n$.

$$
\log \left(M_{n+1}\right)<(n+1)^{2} / 4+(n+1)+2 \log n+4 n^{31 / 32} \log n \text {. }
$$

From the lower bound on $X_{n+1}$ we get $\log \left(M_{n+1} / X_{n+1}\right)<-n / 4$. 
(13) We obtain diagrams in $N(V)$ by considering two cases. In the first case, we suppose that not both of the inequalities $(n+1) / 2-\log n<\left|S_{2}\right|<(n+1) / 2$ $+\log n$ hold. This gives a number $N_{n+1}^{\prime}$ of choices with

$$
\begin{aligned}
\log \left(N_{n+1}^{+}\right) & <2(n+1)+1-\left|S_{2}\right|+\left|S_{2}\right|\left(n+1-\left|S_{2}\right|\right) \\
& <\frac{(n+1)^{2}}{4}+\frac{3(n+1)}{2}-\frac{1}{2}(\log n)^{2} .
\end{aligned}
$$

In the second case, we assume that

$$
\frac{n+1}{2}-\log n<\left|S_{2}\right|<\frac{n+1}{2}+\log n,
$$

and that either

$$
\left|S_{i}\right| \geqslant \frac{n+1}{4}+n^{1 / 2} \log n \text { or }\left|S_{i}\right| \leqslant \frac{n+1}{4}-n^{1 / 2} \log n,
$$

for $i=1$ or 3. This gives a number $N_{n+1}^{n}$ of choices with

$$
\begin{aligned}
\log \left(N_{n+1}^{n}\right)= & 2+(n+1)+\log n+(n+1)^{2} / 4 \\
& +\log \left(\begin{array}{c}
{[(n+1) / 2+\log n]} \\
{\left[(n+1) / 4+n^{1 / 2} \log n\right]}
\end{array}\right) \\
& <(n+1)^{2} / 4+3(n+1) / 2-1 / 4(\log n)^{2} .
\end{aligned}
$$

We get

$$
\log \left(\frac{N_{n+1}}{X_{n+1}}\right) \leqslant \log \left(\frac{N_{n+1}^{\prime}+N_{n+1}^{\prime \prime}}{X_{n+1}}\right)<-\frac{1}{6}(\log n)^{2} .
$$

$\log \frac{O_{n+1}}{P_{n}}<\log (n+1)+25 n^{1 / 2} \log n+\log n$

$$
\begin{aligned}
& +\log \left(\left(\begin{array}{c}
{[(n+1) / 2+\log n]} \\
{\left[(n+1) / 4+n^{7 / 8}\right]}
\end{array}\right)\right. \\
& \quad+2^{\left.(n+1) / 4+n^{1 / 2} \log n+1\left(\begin{array}{c}
{\left[(n+1) / 4+n^{1 / 2} \log n\right]} \\
{\left[(n+1) / 8+n^{7 / 8}\right]}
\end{array}\right)\right)} \\
& <\frac{n}{2}-\frac{1}{10} n^{3 / 4}
\end{aligned}
$$

7. Proof of corollary. For a set $V$ of $n$ vertices, let $Y(V)$ be the class of diagrams $P$ such that $P=L_{1} \vee L_{2} \vee L_{3}$, where $L_{1}, L_{2}, L_{3}$ are the levels of $P$. 
Let $Y_{n}=|Y(V)|$. We obtain all diagrams in $Y(V)$ by first choosing $L_{1}$, then $L_{2}$ and then connecting $L_{2}$ to $L_{1}$ and $L_{3}$ to $L_{2}$. There are exactly $\left(2^{\left|L_{2}\right|}-1\right)$ ways to connect each vertex in $L_{3}$ to $L_{2}$ (there must be at least one connection since $L_{3}$ is a level), and exactly $\left(2^{\left|L_{1}\right|}-1\right)$ ways to connect each vertex of $L_{2}$ to $L_{1}$. This gives

$$
Y_{n}=\sum_{i=1}^{n}\left(\begin{array}{c}
n \\
i
\end{array}\right) \sum_{j=1}^{n-i}\left(\begin{array}{c}
n-i \\
j
\end{array}\right)\left(2^{i}-1\right)^{j}\left(2^{j}-1\right)^{n-i-j}
$$

Since $Y(V) \geqslant Q(V)$, we have

$$
Y_{n}<P_{n}=(1+O(1 / n)) Q_{n} \leqslant(1+O(1 / n)) Y_{n} .
$$

Thus $P_{n}=(1+O(1 / n)) Y_{n}$, and the corollary is proved.

\section{REFERENCES}

1. K. K.-H. Butler, The number of finite partially ordered sets, Notices Amer. Math. Soc. 18 (1971), 1092. Abstract \#71T-A250.

2. S. D. Chatterji, The number of topologies on $n$ points; Kent State University, NASA Technical Report, 1966.

3. L. Comptet, Recouvrements, bases de filtre et topologies d'un ensemble fini, C. R. Acad. Sci. Paris Sér. A-B 262 (1966), A 1091-A 1094. MR 34 \#1209.

4. J. W. Evans, F. Harary and M. S. Lynn, On the computer enumeration of finite topologies, Comm. ACM 10 (1967), 295-298.

5. D. Klarner, The number of graded partially ordered sets, J. Combinatorial Theory 6 (1969), 12-19. MR 38 \#4333.

6. - The number of classes of isomorphic graded partially ordered sets, $\mathrm{J}$. Combinatorial Theory 9 (1970), 412-419. MR 42 \#2989.

7. D. Kleitman and B. Rothschild, The number of finite topologies, Proc. Amer. Math. Soc. 25 (1970), 276-282. MR 40 \#7157.

8. V. Krishnamurthy, On the number of topologies on a finite set, Amer. Math. Monthly 73 (1966), 154-157. MR 34 \#1208.

9. A. Shafaat, On the number of topologies definable on a finite set, J. Austral. Math. Soc. 8 (1968), 194-198. MR 37 \#1263.

DEPARTMENT OF MATHEMATICS, MASSACHUSETTS INSTITUTE OF TECHNOLOGY, CAMBRIDGE, MASSACHUSETTS 02139

DEPARTMENT OF MATHEMATICS, UNIVERSITY OF CALIFORNIA, LOS ANGELES, CALIFORNIA 90024 